\section{Cahiers de Narratologie}

Analyse et théorie narratives

$10.1 \mid 2001$

La voix narrative

\title{
L'ambiguïté narrative dans Clone de Julio Cortàzar
}

\author{
Adriana Castillo-Berchenko
}

\section{OpenEdition}

\section{Journals}

Electronic version

URL: http://journals.openedition.org/narratologie/6950

DOI: 10.4000/narratologie.6950

ISSN: 1765-307X

\section{Publisher}

LIRCES

\section{Printed version}

Date of publication: 1 January 2001

Number of pages: 263-272

ISBN: 2914561032

ISSN: 0993-8516

\section{Electronic reference}

Adriana Castillo-Berchenko, "L'ambiguïté narrative dans Clone de Julio Cortàzar", Cahiers de Narratologie [Online], 10.1 | 2001, Online since 27 October 2014, connection on 23 February 2021. URL: http://journals.openedition.org/narratologie/6950 ; DOI: https://doi.org/10.4000/narratologie.6950 


\title{
L'AMBIGUÏTÉ NARRATIVE DANS « CLONE » DE JULIO CORTÁZAR.
}

\author{
Adriana CASTILLO-BERCHENKO \\ Université de Provence
}

«Clone », nouvelle du recueil Queremos tanto a Glenda (1981) du narrateur argentin Julio Cortázar, propose un modèle narratif qui surprend par son origina-

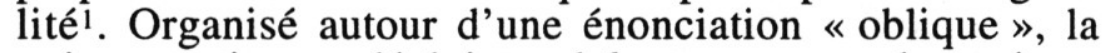
voix narrative se déploie ondulante avec un dynamisme extrême qui peut parfois dérouter ou désorienter le lecteur. $\mathrm{Un}$ « Je » ou un « Tu », un « Il » ou un «Vous », un « Moi », un «On» ou un «Nous» se côtoient dans ce récit se mélangeant, s'entrelaçant, virevoltant inlassablement jusqu'à fusionner avec fougue et élégance. Ainsi, ces jeux de l'énonciation confèrent au récit une étrange plurivocité qui fait de "Clone » une histoire à clés, une fable énigmatique assez inquiétante. D'où vient l'ambiguïté, cette condition amphibologique tenace qui semble se dégager du texte? Comment détermine-t-elle une structure narrative équivoque et « en miroirs » comme celle-ci ? A travers l'analyse textuelle, on essaiera d'élucider ces questions. On s'arrêtera sur le problème de la voix narrative pour tenter de saisir par la suite sa nature profonde. On s'intéressera enfin aux effets qu'une telle modalité d'écriture provoque chez le récepteur.

Un lecteur complice et participatif, voilà le récepteur idéal des nouvelles de Cortázar : quelqu'un qui décrypte avec aisance les règles du jeu narratif et esthétique que chacune de ses histoires impose. "Clone » évidemment n'échappe pas à ce postulat. Oscillant, méandreux, ce récit met en place un groupe de chanteurs de madrigaux

1 Julio CORTAZAR, Queremos tanto a Glenda, Madrid, Alfaguara, 1981, pp. 105-127. 
d'exception. Ils sont huit et vivent la dernière étape d'une longue tournée internationale et d'une fructueuse existence de triomphes. À présent, un sentiment de malaise fragilise le groupe. Les doutes, la suspicion, les craintes, le silence fissurent lentement un esprit de corps jusqu'alors soudé. C'est que l'un des membres de l'octuor a brisé le pacte implicite qui les liait et transgressé de la sorte les règles du jeu les unissant.

Cette intrigue, certes simplifiée, laisse entrevoir cependant l'essentiel des forces en action au cœur de l'histoire. Tout se joue en effet dans les rapports de force du groupe de chanteurs en tant que corps, un tout uni, dense, un "clone " et les individus, chaque être, chaque madrigaliste l'intégrant. Ainsi donc, le titre ne s'érige ici que comme une énigme à moitié, située de surcroît au centre d'une fable en paliers fort dense, enchevêtrée, complexe. Comme l'affirme l'un des piliers de l'octuor: «Cantábamos y vivíamos como uno solo [...] es cierto, el canto y la vida y hasta los pensamientos eran una sola cosa en ocho cuerpos $»^{2}$. C'est justement là, en conséquence, dans la fusion intime du «chant », de la «vie» et de la "pensée " qu'être un "clone » a pris du sens pour le groupe. La pratique exceptionnelle d'une conception de l'art et de la vie comme un tout inséparable devient de surcroît l'expérience troublante de la multiplicité dans l'unité. C'est ainsi que les chanteurs ont trouvé un équilibre, une cohésion sans ruptures. Mais voici qu'à présent, l'état de grâce se dégrade et que le "clone » tant privilégié se désagrège. Cette détérioration constitue l'essentiel d'un récit qui sous la forme d'un texte et d'un paratexte, l'observe et la décortique à partir des points de vue de ceux qui la subissent ${ }^{3}$. C'est alors que la nature de l'énonciation devient signifiante.

2 Ibid., p. 110.

3 Le texte porte le titre de "Clone» et s'organise en seize séquences (pp. 105-121) à l'apparence très fluide presque sans discours directs. Le paratexte porte le titre " Nota sobre el tema de un rey y la venganza de un príncipe» (pp. 122-127). Il se présente comme un écrit autonome apparemment sans rapport avec le texte le précédant. Cette ambiguité (voulue ?) de la part de Cortázar peut 
L'instance narrative dans «Clone » se configure sous le signe du doute, de la confusion et du non engagement. Une série de voix qui s'entrecroisent fait avancer le récit dans des phrases à l'apparence limpide et parfaite : «Empiezan a sospechar, lo sé, y qué voy a hacer si es como una enfermedad [...] Ah, si otro de nosotros dirigiera, si Karen o Roberto dirigieran para que yo pudiera diluirme en el conjunto [...] Ahí como lo ves está siempre ahora, dice Paola, [...] soñando despierto, en mitad del más jodido de los Gesualdos, cuando hay que medir al milímetro para no irse al corno, justo entonces se te queda como en el aire, carajo. Nena, dice Lucho, las mujeres bien no dicen carajo. Pero con qué pretexto hacer el cambio, hablarle a Karen o a Roberto ${ }^{4}$. Ces voix, leurs discours, appartiennent aux chanteurs de l'octuor. Ils échangent des points de vue. Ils discutent sur les faits de leur quotidien d'artistes en tournée. Le contenu des messages est fragmentaire, les interlocuteurs se reconnaissent dans une camaraderie et une complicité qui favorise l'implicite. On devine alors sous la phrase explicitée, le non-dit, un silence éloquent et voulu. Progressivement, ces échanges faits de bribes de conversation ou de pensées inabouties s'entrelacent et composent de la sorte une grille de sens qui se complète. L'échange discursif, puisqu'on est confronté à des énoncés individuels, devient alors un mélange expressif qui oscille, virevoltant autour d'un conflit d'ordre professionnel et existentiel qui se dessine lentement. A ce stade, plus le récit progresse, plus les voix se répondent et plus l'impression d'une voix collective s'impose. La cacophonie envahirait alors le texte ? Absolument pas. C'est qu'entraînés dans leur propre mouvement interne, ces voix ont évolué, passant du simple échange au mélange étroit pour se retrouver enfin dans la fusion énonciative généralisée, peut-être même chorale.

Tout cela semble fort paradoxal. D'autant plus que le texte s'étale dans des phrases courtes qui s'inscrivent dans

induire aisément en erreur le lecteur non averti. Les rapports entre texte et paratexte n'étant pas évidents, ce n'est qu'après une lecture attentive et longue réflexion que les liens les unissant cristallisent.

4 Cortázar, op. cit., p. 109. 
des paragraphes lisses et harmonieux organisés en blocs (un tout, un clone) constituant de la sorte les seize séquences du récit. L'apparence de fluidité est néanmoins trompeuse car, d'une manière discrète, masquée et très subtile, ces séquences sont véhiculées par une voix extérieure qui de fait contrôle fermement le tout. Cette deuxième donnée de la composition de l'instance narrative (la première étant la série de voix qui s'expriment au sein du texte) joue un rôle décisif dans le tissage de sens proposé par les différentes voix individuelles.

Très diffuse, adoptant volontairement une posture énonciative en retrait, presque in absentia, cette voix que l'on peut désigner comme une voix off veille en permanence sur la textualité. Certes, la découvrir dans son action n'est pas aisé tant elle se déguise pour devenir invisible et insaisissable. Elle règne pourtant sur treize des seize séquences constituant la nouvelle. Il semble alors qu'elle est bien là pour accomplir une tâche, pour laisser une trace et engendrer du sens. De fait, si la voix off avance fantomatique et privilégie un statut quasiment in absentia, elle le fait pour mieux souligner que les autres voix, celles des chanteurs de madrigaux, sont bel et bien des voix in praesentia.

En effet, on remarque aisément que l'important dans la cristallisation de l'histoire c'est de mettre en avant les arguments, les points de vue du groupe. La voix off cantonnée dans une troisième personne singulière ou plurielle énonce, oriente et subtilement ondule entre les voix individuelles. De la sorte, et sans crier gare, elle pénètre dans les personnages, s'approprie leurs discours et profitant des effets de l'intériorisation bouscule et change les lignes du récit. La phrase limpide reste égale à ellemême mais le jeu de sens du discours narratif est, lui, modifié. Il est devenu oblique, ambigu, inquiétant: "Porque la venganza es un arte, sus formas buscarán necesariamente las circunvolociones que la vuelven más sutilmente bella. "Es curioso" piensa Mario "que alguien capaz de concebir el universo sonoro que surgía de los madrigales se vengara tan crudamente [...] mira a Paula que trabaja y repite un pasaje [...] Le sonríe [...] Sabe muy bien 
por qué Paola ha vuelto a hablar de Gesualdo [...] Sete, le dice, no marques tanto sete, Paolita, la sed se la siente con más fuerza si dices suavemente la palabra $»^{5}$.

Par quels artifices la voix off subjugue les autres ? La révision attentive de l'ensemble des séquences met à jour son action. L'histoire de "Clone ", on le sait, se déploie en seize séquences. Les trois premières organisent le jeu énonciatif dans une combinaison harmonieuse de la voix des personnages. Il est clair à ce moment-là que la voix off dirige et que les autres assument docilement son jeu. Dans les séquences quatre et cinq une modification se produit : les voix des personnages imposent leurs discours sur une voix off presque absente. De fait, celle-ci a autorisé ces discours grâce aux jeux de focalisation interne. Les séquences six et sept, en revanche, accentuent et donnent toute sa puissance à la voix off qui devient ici le maître du récit. La huitième séquence, cœur de l'histoire, s'organise en discours direct autonome à l'état pur. Seules les voix des personnages s'expriment dans un entrecroisement serré d'arguments, d'attaques et de contre-attaques. La voix off est absente. Les huit dernières séquences avancent dans un rythme haletant. Un climax dans l'avantdernière séquence prépare un dénouement brutal, surprenant et énigmatique. Dans ces huit séquences la voix off est toujours présente mais en retrait ; elle dirige habilement le jeu des voix individuelles qui toutes (sauf une) ont droit au discours.

Ainsi donc, sous l'égide de la voix off, les discours individuels se croisent et se superposent, se multiplient et se diversifient le long des séquences. On remarque de ce fait un crescendo énonciatif qui va de pair avec l'accumulation des événements annonçant l'imminence d'une issue fatidique $^{6}$. Et l'on voit bien enfin que dans cet ensemble des séquences, c'est surtout l'énonciation en retrait et en

\section{Ibid., pp. 117-118.}

6 L'histoire est close par la disparition de Franca, l'une des sopranos de l'ensemble. Elle est la cause "visible " de l'implosion du groupe. Sa disparition reste une énigme mais elle entraîne la chute générale des musiciens. Des huit membres de l'octuor, Franca est le seul personnage qui n'a pas droit au discours. 
sourdine de la voix off qui est l'organisatrice du jeu. En ce sens, le dénouement s'affirme comme une éclatante polyphonie généralisée exprimant simultanément la fusion des voix et l'implosion affolée du clone atomisé, morcelé à jamais. Pourquoi donc tout cela ?

A l'intérieur de la trame, vengeance et passion coupable composent un duo permanent se conjuguant tout d'abord au niveau des interprètes des madrigaux : Sandro, l'un des ténors et directeur artistique, Franca, soprano et femme de Mario la basse du groupe. Ils vivent un rapport passionnel et conflictuel dont les cinq autres chanteurs sont témoins. Cette relation triangulaire répercute en outre dans le répertoire des madrigalistes. L'une des raisons de leur succès international est l'interprétation des Madrigali du compositeur de la Renaissance, Carlo Gesualdo ${ }^{7}$. Ce musicien génial était aussi un prince et un mari jaloux. Devant la preuve de l'adultère dont il a été la victime, il décrète la mort des coupables. Il compose plus tard les Madrigali ${ }^{8}$. On voit bien alors que par un effet d'analogies, la trame se tisse dans une composition en miroirs dont les reflets à niveaux divers s'interpellent et correspondent. De ce fait, à la passion coupable des amants illégitimes répond la cruauté vengeresse d'un mari trompé. Certes, la confrontation a lieu dans des temps et des espaces différents, les protagonistes sont eux aussi dans des sphères opposées. Rien n'y fait. La passion contemporaine ressemble fort à celle de la Renaissance quatre siècles auparavant, la

7 Carlo Gesualdo, prince napolitain de Venosa (1560-1613) fut l'un des musiciens les plus importants de la fin de la Renaissance italienne. En 1590, il tua ou fit tuer devant lui sa femme et son amant. Entre 1594 et 1613, il composa cinq Livres de Madrigali.

8 Dans ses Madrigali, Gesualdo se révèle comme un musicien innovateur presque révolutionnaire. Son discours musical met en avant la recherche d'une liberté de langage et d'une intensité d'expression toujours plus grandes dont le choix de textes poétiques (Le Tasse, de préférence) joue un rôle important. Son art explose dans les derniers madrigaux (Livres IV et V). Ils révèlent la violence de son tempérament. Il ne recule devant aucune étrangeté harmonique, ne craint aucun contraste rythmique pour exprimer même brutalement son cri intérieur. 
douleur des époux délaissés, musiciens et témoins de leurs échecs, aussi.

Les correspondances ainsi établies dévoilent les règles du jeu au sein de la trame. Les forces de l'amour et de la mort, de la haine et de la vengeance sont ici le ressort de la musique. Elle est à la fois leur lien et leur porteuse. Aussi bien de ce côté de la réalité que de l'autre, au XXe ou au XVIe siècles, elle rassemble, unit et justifie l'être et le paraître des faits et des personnages. Tout s'explique alors et le jeu du chassé-croisé de l'instance narrative ainsi que les polyphonies qu'il engendre deviennent enfin clairs et légitimes.

La nature polyphonique de l'énonciation est justement la raison de la « Nota sobre el tema de un rey y la venganza de un príncipe » paratexte de "Clone ». En ce sens ce titre à double signifiant peut étonner. C'est que poussé par le besoin de justifier les difficultés de son histoire, Cortázar s'explique sur ses origines. "Clone » affirme-t-il est une œuvre motivée par l'audition de L'offrande musicale, composition de J.S. Bach. On découvre ainsi avec étonnement que beaucoup plus déterminant que les Madrigali de Gesualdo, jouant jusqu'alors le rôle de source nourricière du récit, L'offrande musicale de Bach est le macrotexte fournissant le modèle des polyphonies 9 . L'objectif du paratexte devient finalement clair. Cortázar l'a rédigé dans l'espoir de rendre lisible l'histoire. Hélas, aussi ambigu et oblique

${ }^{9}$ L'offrande musicale (Das musikalische opfer) de 1747 est une œuvre de Bach dont l'origine fut la rencontre à Postdam du roi du Prusse, Frédéric II. Après une série d'improvisations de Bach, le roi lui propose un thème de son cru en lui demandant d'improviser sur lui une fugue. Quelques temps plus tard, de Leipzig, Bach expédia au roi une œuvre, L'offrande musicale, sur le fameux thème royal. Sur ce thème, Bach compose une fugue à trois voix (Ricercare 1), six canons, une fugue canonique, une sonate en trio en quatre mouvements, trois autres canons et une fugue à six voix (Ricercare 2 ). Ces morceaux, notamment les canons donnent lieu à de nombreuses variations. L'offrande musicale est une œuvre pleine d'humour, d'imagination et de spiritualité. 
que le texte le précédant il fixe les sens et simultanément, il les confond.

De fait, l'organisation textuelle en seize séquences, l'engagement narratif dans une double instance des voix qui se nouent et se dénouent, cette écriture à la fois classique et baroque (nette et confuse) de "Clone " correspond tout à fait à la matrice de l'œuvre de $\mathrm{Bach}^{10}$. Mais là où la ressemblance devient troublante c'est justement dans le traitement du motif «el tema de un rey » qui, tout comme celui de "la venganza de un príncipe », marquent de leur empreinte l'histoire et brisent de la sorte les conventions du temps et de l'espace. «La venganza de un príncipe » habite, on le sait, la trame. Le «tema de un rey » par contre, renforce (tout comme il le faisait dans L'offrande musicale de Bach) le motif structurant de l'histoire, celui des rapports troubles entre l'art et la passion, la musique et le pouvoir, la création et le créateur. Ces rapports conflictuels sont véhiculés dans tous les sens dans "Clone" : au niveau des événements, dans les rapports des chanteurs entre eux et dans leurs rapports avec les compositeurs qu'ils interprètent, notamment avec Gesualdo dont le drame de trahison et vengeance correspond avec celui de Mario, la basse du groupe. En effet, de même que Gesualdo décréta la mort violente de sa femme et son amant, Mario décrète à son tour, la disparition de Franca, la mort artistique de Sandro et du groupe des chanteurs de madrigaux. Mais si la mort des coupables déclenche chez Gesualdo la pulsion créatrice et la naissance d'un chef d'œuvre, dans le cas de Mario, la vengeance est destructrice et définitive. De ce point de vue-là, "Clone » est aussi une histoire d'anéantissement, de disparition et de mort.

10 Les seize séquences de "Clone" correspondent aux seize morceaux de L'offrande musicale de Bach. Le Ricercare 1 (fugue à trois voix) correspond à la séquence première avec une énonciation entrecroisée à trois voix de même que le Ricercare 2 (fugue à six voix) correspond à la seizième séquence. La sonate en trio à quatre mouvements cristallise, elle, dans les neuvième, dixième, onzième et douzième séquences. Les canons, tous de nature diverse, donnent lieu à de nombreuses variations dans les autres séquences. 
Plus frappants encore sont les rapports concernant la forme dans "Clone ». Au niveau de l'organisation textuelle, par exemple, les seize séquences de la nouvelle équivalent aux seize morceaux de L'offrande musicale de Bach. Beaucoup plus raffinés enfin sont les rapports qui concernent l'énonciation. Elle reproduit, de fait, nombre de combinaisons polyphoniques appartenant à L'offrande musicale et le fait avec des variantes subtiles presque insaisissables. C'est là enfin que l'on peut mesurer le talent de Cortázar. En effet, les harmonisations des voix qui engendrent les polyphonies de «Clone » reproduisent de manière délicate l'éventail harmonique des canons, ricercares, fugues et sonates de L'offrande musicale. En ce sens, la voix off, extérieure, dont la posture in absentia joue avec les voix des chanteurs in praesentia, accomplit parfaitement le rôle du continuo dans L'offrande musicale. Comme lui, la voix off organise, oriente, donne le ton et accompagne le jeu des voix individuelles qui, elles, traduisent le jeu instrumental de l'original en jeu vocal à la manière des madrigaux gesualdiens ${ }^{11}$. Ainsi se combinent les voix des ténors, de la basse, des sopranos, de la mezzo et du baryton. Par ce procédé d'hybridation parfaite, Cortázar réussit un véritable tour de force.

Et le lecteur dans ce jeu, quel est son rôle ? Le récepteur accepte ou refuse. Il peut recevoir "Clone» comme un théorème ambigu susceptible de plusieurs démonstrations. En ce sens, il peut se satisfaire d'une lecture purement événementielle et se dire qu'en fin de compte Cortázar fictionnalise ici, comme il l'a souvent fait, les difficultés de l'être ou qu'il privilégie, encore une fois, les signes inquiétants d'un quotidien trompeur. Il peut se

11 Si la voix off joue le rôle du continuo se déguisant comme une présence-absence dans la phrase narrative, les voix individuelles se combinent dans un jeu hybride très riche issu des combinaisons inspirées par L'offrande musicale de Bach et par les Madrigali de Gesualdo. Chez Cortázar, il ne faut pas l'oublier tout est question de voix : la voix de la musique et la voix narrative fusionnent en conséquence de manière totale, absolue. 
dire aussi que Cortázar a voulu raconter l'histoire d'un couple ou bien d'un groupe en crise. Les possibilités de lecture de «Clone » sont décidément multiples et variées. On a ici un authentique jeu de kaléidoscope admettant même les lectures extrêmes: histoire de doubles à répétition, récit de possession ou de vampirisme et autres encore. Un lecteur curieux, complice comme Cortázar les aimait, se sentira par contre beaucoup plus attiré par une lecture «texte-paratexte». Il interpellera l'énigmatique paratexte et préférera certainement une lecture-aventure ou une lecture-enquête qui, elle, lui permettra au mieux de déceler le labyrinthe passionnant et passionné de l'écriture chez Cortázar. 